\title{
Conditioned Medium from Bone Marrow Mesenchymal Stem Cells Transiently Retards Osteoblast Differentiation by Downregulating Runx2
}

\author{
Jing Sun Huifang Zhou Yuan Deng Yidan Zhang Ping Gu Shengfang Ge \\ Xianqun Fan
}

Department of Ophthalmology, Ninth People's Hospital, Shanghai Jiaotong University School of Medicine, Shanghai, PR China

\section{Key Words}

Bone marrow mesenchymal stem cell $\cdot$ Osteoblast

differentiation · Osteogenesis $\cdot$ Conditioned medium

\begin{abstract}
Mesenchymal stem cells (MSCs) are attractive candidates for cell therapy and regenerative medicine because of their potential for proliferation and multilineage differentiation. MSCs secrete various cytokines, acting as trophic mediators to regulate neighboring cells. Osteoblasts are the cells directly responsible for forming new bone, and they are the final target of many osteogenic regulators. However, the induction effect of MSCs on osteoblasts is still unknown. In this study, we isolated osteoblasts from rat calvariae and investigated their proliferation and differentiation under induction of varied concentrations of MSC-conditioned medium (MSC$\mathrm{CM}$ ). Cells in the MSC-CM groups showed a reduction in cell proliferation at 3-6 days, and a decrease in the expression of osteocalcin and osteopontin at 3 days, with low levels of alkaline phosphatase activity. The expression of osteogenic markers went back to normal at 7 days. In order to evaluate the molecular mechanisms underlying this suppression, levels of two osteoblastic transcription factors, runt-related transcription factor 2 (Runx2) and osterix (Osx), were detected at both mRNA and protein levels. The results indicated
\end{abstract}

that MSC-CM significantly inhibited Runx2 expression in a concentration-dependent manner. However, the effect was not due to the inhibition of Osx, for Osx was not significantly altered. This work demonstrates that MSCs may suppress osteoblast proliferation and transiently retard osteoblast differentiation by downregulating Runx2. These results highlight the need to take into account the paracrine effect of MSCs when using them in regenerative medicine for the repair of bone defects.

Copyright $\odot 2012$ S. Karger AG, Base

\section{Introduction}

Mesenchymal stem cells (MSCs) constitute a relatively rare population, primarily in adult bone marrow (BM) [Deans and Moseley, 2000], but they can also be obtained from some other adult and fetal tissues [Erices et al., 2000; Gronthos et al., 2001]. They are multipotent cells capable of differentiating into various lineages, including osteoblasts, chondrocytes, adipocytes, and even skin keratinocytes, under appropriate induction conditions in vitro [Pittenger et al., 1999; Majumdar et al., 2000; Chun-mao

\section{J. Sun and H. Zhou were equal contributors to this work.}




\begin{tabular}{ll}
\hline Abbreviations used in this paper \\
\hline$\alpha-$ MEM & $\begin{array}{l}\alpha \text {-minimal essential medium } \\
\text { alkaline phosphatase }\end{array}$ \\
BMP & bone marrow \\
BMPs & bone morphogenetic proteins \\
DAPI & 4,6-diamino-2-phenyl indole \\
DMEM & Dulbecco's modified Eagle's medium \\
DMSO & dimethyl sulfoxide \\
FBS & fetal bovine serum \\
FGF-2 & fibroblast growth factor 2 \\
IGF-1 & insulin growth factor 1 \\
IL-1 $\beta$ & interleukin-1 beta \\
IL-6 & interleukin-6 \\
MSC-CM & mesenchymal stromal cell-conditioned medium \\
MSCs & mesenchymal stromal cells \\
Msx2 & msh homeobox 2 \\
MTT & methyl thiazolyl tetrazolium \\
OCN & osteocalcin \\
OPN & osteopontin \\
Osx & osterix \\
PBS & phosphate-buffered saline \\
pNPP & p-nitrophenyl phosphate \\
Runx2 & runt-related transcription factor 2 \\
SD & Sprague-Dawley \\
TNF- $\alpha$ & tumor necrosis factor alpha \\
\hline & \\
&
\end{tabular}

et al., 2007]. Also, MSCs can easily be expanded for more than 50 passages while retaining their multilineage differentiation potential in culture. Their regenerative and reparative potential makes them attractive candidates for cell therapy and regenerative medicine. In bone tissue engineering, MSCs are usually combined with a biomaterial scaffold as seeding cells to treat bone defects [Horwitz, 2006; Kasten et al., 2006; Caplan, 2007; Xiao et al., 2011; Zhou et al., 2011].

MSCs secrete many cytokines and growth factors, which may play important roles in a variety of physiological activities. They have the capability to promote or suppress the expansion of specific kinds of T cells, and perform an immune regulatory function [Aggarwal and Pittenger, 2005; Guo et al., 2009]. Some protective factors secreted by MSCs act as a primary matrix for tissue regeneration during inflammation and tissue injuries [Hass et al., 2011]. MSCs take on an anti-inflammatory role by decreasing expression of the inflammatory cytokines interleukin-1 beta (IL-1 $\beta$ ), interleukin-6 (IL-6), and tumor necrosis factor alpha (TNF- $\alpha$ ) [Guo et al., 2007]. Some of the bioactive factors secreted by MSCs could suppress the local immune system, inhibit fibrosis (scar formation) and apoptosis, and enhance angiogenesis, acting as trophic mediators [Caplan, 2006]. All of the functions underlie a significant potential for the clinical use of MSCs.

Osteoblasts are responsible for the formation of new bone by synthesizing mineralized bone matrix under physiological conditions. They are very sensitive to their environment. Since osteoblasts are directly associated with bone synthesis, the proliferation and differentiation of osteoblasts is always an attractive issue in the physiology of bone and in the study of bone repair [Feng et al., 2011]. It has been reported that various types of stimulation, either mechanical or biochemical, may influence the proliferation and differentiation of osteoblasts [KadowRomacker et al., 2009; Fei, 2011]. However, the induction effect of MSCs on osteoblasts is still unknown.

In the present study, osteoblasts isolated from rat calvariae were cultured in conditioned medium (CM) prepared from MSCs. The characteristics of osteoblasts following induction were investigated. The proliferation of osteoblasts was assessed using methyl thiazolyl tetrazolium (MTT) assay and cell cycle analysis, and then the induction effect on differentiation was investigated by measurement of the alkaline phosphatase (ALP) expression level. Using real-time polymerase chain reaction (qPCR), Western blotting, and immunofluorescent staining analysis, the effects of MSC-CM on the expression levels of osteocalcin (OCN), osteopontin (OPN), runt-related transcription factor 2 (Runx2), and osterix (Osx) were investigated to gain a deeper insight into the molecular mechanism involved. Given that the osteoblast is the only cell in the body that is capable of producing new bone, the information from this study may provide clues that will prove useful for bone defect treatment, contributing to the development of a strategy for perfect bone tissue engineering.

\section{Materials and Methods}

Isolation, Culture, and Identification of MSCs

This study was permitted under the Law of the People's Republic of China on the Protection of Wildlife, and the protocol was approved by the Animal Ethics Committee of the Ninth People's Hospital, Shanghai Jiaotong University School of Medicine. Sprague-Dawley (SD) rats were obtained from the Medical Institute Animal Center of Shanghai Jiaotong University, China. BMSCs were isolated and expanded using previously reported methods [Xiao et al., 2011]. BM was harvested from SD rats aged 8-10 weeks. After euthanasia, the femurs and tibias were removed from the body, and the BM was flushed out of the bones using a syringe. The cells collected were centrifuged, resuspended in $\alpha$ minimal essential medium ( $\alpha$-MEM; Gibco, USA) supplemented with $10 \%$ fetal bovine serum (FBS; Gibco), $100 \mathrm{IU} / \mathrm{ml}$ penicillin, 
and $100 \mu \mathrm{g} / \mathrm{ml}$ streptomycin (Sigma-Aldrich, St. Louis, Mo., USA), and plated into $25 \mathrm{~cm}^{2}$ plastic flasks at a density of $1 \times 10^{5}$ cells $/ \mathrm{cm}^{2}$, followed by incubation in a humidified atmosphere with $5 \% \mathrm{CO}_{2}$ at $37^{\circ} \mathrm{C}$. The medium was refreshed every 3 days. When the primary MSCs reached almost 100\% confluence, they were passaged using $0.25 \%$ trypsin with $0.02 \%$ EDTA (Gibco) and plated into Dulbecco's Modified Eagle's Medium (DMEM; Gibco) supplemented with $10 \%$ FBS. At passage 5 of the MSCs, when they reached approximately $90 \%$ confluence, the medium was refreshed; then the CM was collected after $12 \mathrm{~h}$ of incubation, centrifuged at $1,200 \mathrm{rpm}$ for $5 \mathrm{~min}$, and stored at $-80^{\circ} \mathrm{C}$. This supernatant was named MSC-CM.

MSCs were assessed by cytofluorimetric analysis for expression of the typical markers CD29, CD44, and CD90, and absence of the hematopoietic markers CD45, CD34, and CD11b. MSCs at passage 3 were collected and washed with phosphate-buffered saline (PBS) and then resuspended at $3.0 \times 10^{6}$ cells $/ \mathrm{ml}$. Appropriate specific antibodies were added (FITC anti-rat CD90, PE antirat $\mathrm{CD} 29, \mathrm{PE}$ anti-rat $\mathrm{CD} 45$, and $\mathrm{PE}$ anti-rat $\mathrm{CD} 11 \mathrm{~b} / \mathrm{c}$, all from Biolegend, San Diego, Calif., USA; CD34 antibody from Santa Cruz, Calif., USA; mouse anti-rat CD44 from Serotec, Oxford, $\mathrm{UK}$, isotype-matched controls and blank control). After incubation at $4^{\circ} \mathrm{C}$ for $30 \mathrm{~min}$ protected from light, the cells were washed and resuspended and then detected by flow cytometry. Quantitative fluorescence analysis was performed using a FACSCalibur cytometer and CellQuest software program (Becton Dickinson, Franklin Lakes, N.J., USA). The cell number (at least 10,000 cells) versus fluorescence intensity was recorded for each sample.

\section{Isolation, Culture, and Identification of Osteoblasts}

Rat primary osteoblasts were isolated from SD rats $24-48 \mathrm{~h}$ postnatally. The calvariae were dissected and the periosteum completely stripped away; the bones were then cut into small pieces and processed by sequential digestions of $20 \mathrm{~min}$ at $37^{\circ} \mathrm{C}$ in $0.25 \%$ trypsin and $1 \mathrm{~h}$ at $37^{\circ} \mathrm{C}$ in $0.1 \%$ collagenase II (fig. $2 \mathrm{a}, \mathrm{b}$ ). Cells harvested from the first digestion were discarded. After the second digestion, cells were collected and resuspended in DMEM supplemented with $10 \% \mathrm{FBS}, 100 \mathrm{IU} / \mathrm{ml}$ penicillin, and $100 \mu \mathrm{g} / \mathrm{ml}$ streptomycin. They were plated in $25-\mathrm{cm}^{2}$ plastic flasks at a density of $1 \times 10^{5}$ cells $/ \mathrm{cm}^{2}$ and incubated in a humidified atmosphere of $5 \% \mathrm{CO}_{2}$ in air at $37^{\circ} \mathrm{C}$. The medium was refreshed every 3 days. Osteoblasts at passages $2-4$ were used for the experiments described below.

Osteoblasts were identified by ALP and Alizarin red staining. Cells were seeded onto coverslips and incubated overnight to allow cells to adhere. Then ALP staining was performed according to the manufacturer's instructions (Rainbow, Shanghai, China). After 2 weeks of culture in osteogenic induction medium, Alizarin red staining was performed using a Cell Alizarin Red S Kit (Genmed, Shanghai, China) to assess bone nodule formation. Osteoblasts were fixed with $4 \%$ paraformaldehyde for $30 \mathrm{~min}$ and then incubated with the staining solution for $15 \mathrm{~min}$ at room temperature. PBS was used for washing and as the negative control.

\section{Cell Proliferation Assay}

The effect of $\mathrm{CM}$ on osteoblast proliferation was evaluated with MTT [3-(4,5-dimethylthiazol-2-yl)-2,5-diphenyl tetrazolium bromide] assay (Sigma). Osteoblasts were cultured in 96-well plates for up to 6 days with 1:4 medium (1 part CM:4 parts DMEM $+10 \%$ FBS), 1:2 medium, 1:1 medium, or total DMEM + 10\% FBS medium. After the cells were washed with PBS twice, $180 \mu \mathrm{l}$ medium as described above supplemented with $20 \mu 15 \mathrm{mg} / \mathrm{ml}$ MTT (Sigma) solution was added to each well. The plate was incubated at $37^{\circ} \mathrm{C}$ for $4 \mathrm{~h}$ to allow formation of MTT formazan. Then the medium was replaced with $150 \mu$ l dimethyl sulfoxide (DMSO; Sigma) and shaken at room temperature for $15 \mathrm{~min}$ in order to dissolve the formazan. The absorbance measured at a wavelength of 490 nm (ELX Ultra Microplate Reader; Bio-tek, Winooski, Vt., USA) was plotted against days of culture.

\section{Cell Cycle Assay}

Osteoblasts were cultured in 6-well plates for 3 days with 1:4 medium (1 part CM:4 parts DMEM + 10\% FBS), 1:2 medium, 1:1 medium, and total DMEM + 10\% FBS medium, respectively. Then the cells were trypsinized and fixed with $75 \%$ ethanol at $4^{\circ} \mathrm{C}$ overnight. The next day, the cells were centrifuged and washed with PBS twice and then incubated with PI/RNase Staining Buffer (BD Biosciences) for $30 \mathrm{~min}$. The proportion of cells in each phase of the cell cycle was detected by flow cytometry (FACSCalibur; Becton Dickinson).

\section{Quantitative ALP Activity Assay}

For the determination of ALP activity, osteoblasts were seeded in 24-well plates and fed different culture media for 3 and 6 days. Cells were then rinsed twice with PBS and lysed with $0.1 \%$ TritonX-100 in $10 \mathrm{~mm}$ Tris- $\mathrm{HCl}$ ( $\mathrm{pH}$ 9.0). ALP activity was determined using p-nitrophenyl phosphate (pNPP; Sigma) as the substrate, as described previously [Sun et al., 2006]. Each 100- $\mu$ l sample was mixed with $100 \mu \mathrm{l} \mathrm{pNPP}(1 \mathrm{mg} / \mathrm{ml})$ in $1 \mathrm{M}$ diethanolamine buffer $\left(0.5 \mathrm{mM} \mathrm{MgCl}_{2}, \mathrm{pH} 9.8\right)$ and incubated at $37^{\circ} \mathrm{C}$ for $15 \mathrm{~min}$. Then $12 \mu \mathrm{l}$ of $3 \mathrm{~N} \mathrm{NaOH}$ was added to stop the reaction. The absorbance was measured at a wavelength of $405 \mathrm{~nm}$. At the same time, the total protein content of each well was determined in aliquots of the same samples by the Bradford method using the Bio-Rad protein assay kit (Bio-Rad, Hercules, Calif., USA), absorbance was measured at $630 \mathrm{~nm}$, and the concentration was calculated according to a series of BSA (Sigma) standards. ALP activity was expressed as absorbance at $405 \mathrm{~nm}$ (OD value) per milligram of protein. All experiments were conducted in triplicate.

\section{RNA Extraction, cDNA Synthesis, and Real-Time RT-PCR}

Osteoblasts were seeded in 6-well plates at an initial density of $15 \times 10^{4}$ cells/well and fed with various mixtures of culture medium as described above for 3 days. Total RNA was extracted using Trizol reagent (Invitrogen, Carlsbad, Calif., USA). Total RNA $(1,500 \mathrm{ng})$ was reverse transcribed to cDNA with a PrimeScript 1st Strand cDNA Synthesis kit (Takara, Tokyo, Japan) following the manufacturer's instructions. Real-time PCR analysis was performed as previously described [Zhang et al., 2010]. The cDNA was used as the template for real-time PCR (qPCR) amplification using primers of ALP sense: $5^{\prime}$-CCT TGA AAA ATG CCC TGA AA, and antisense: $5^{\prime}$-CTT GGA GAG AGC CAC AAA GG; OCN sense: 5'-TGA GGA CCC TCT CTC TGC TC, and antisense: 5'AGG TAG CGC CGG AGT CTA CC; Runx2 sense: 5'-GCC GGG AAT GAT GAG AAC TA, and antisense: 5'-GGA CCG TCC ACT GTC ACT TT; Osx sense: ATC TGA GTG AGC CGG CCT GAG AG, and antisense: 5'-AAG GGA GCT GGG TAG GCG TCC; GAPDH sense: CTC ATG ACC ACA GTC CAT GC, and antisense: 5'-TTC AGC TCT GGG ATG ACC TT. SYBR quantitative real-time PCR was performed using an ABI PRISM 7900 HT se- 
quence detector (Applied Biosystems, Foster, Calif., USA) according to the manufacturer's instructions (Takara), and the data were normalized against the CT (threshold cycle) of the GAPDH control.

\section{Western Blot Analysis}

Total protein extracts of cultured cells in each group were prepared using nuclear and cytoplasmic extraction reagents (Pierce, Rockford, Ill., USA) as previously described [Zhang et al., 2011]. The protein concentration was measured using BCA Protein Assay Reagent (Pierce). Cell lysates $(30 \mu \mathrm{g})$ were electrophoresed on $12 \%$ polyacrylamide slab gels and transferred to the polyvinylidene difluoride membranes. The membrane was incubated with primary antibodies of OCN (Millipore, Billerica, Mass., USA), OPN (Abcam, Cambridge, Mass., USA), Runx2 (Abcam), Osx (Abcam), and $\beta$-actin (Sigma), respectively. Secondary antibodies were labeled with IRDyes. Signals were visualized using an Odyssey Infra-red Imaging System (LI-COR Biosciences, Lincoln, Nebr., USA). $\beta$-Actin was used as the internal control to normalize the loading materials. We first read the gray value of every band with Odyssey and then calculated the ratio of target protein $/ \beta$-actin. We calculated the value of the ratio of CM groups/the ratio of the control group as the ratio of the control group was taken for 1 . Data of three separate experiments were used for calculation, and the obtained values were graphed.

\section{Immunofluorescent Staining}

Cells in each group were fixed in 4\% paraformaldehyde in PBS for $15 \mathrm{~min}$ and then blocked with 10\% horse serum (Sigma) for $1 \mathrm{~h}$. After permeabilization with $0.3 \%$ Triton X-100 (Sigma), the cells were incubated with primary antibodies in $4^{\circ} \mathrm{C}$ overnight and Dylight 488 secondary antibody (Sigma) for $1 \mathrm{~h}$ at room temperature. 4,6-Diamino-2-phenyl indole (DAPI) (Sigma) was used to stain nuclei.

\section{Statistical Analysis}

All experiments were repeated 3 times and the measurements expressed as means \pm standard deviations. Data were analyzed using Student's two-tailed t test to compare the means of two groups and one-way ANOVA for comparison of the means of more than two groups, assuming equal variance, using SPSS Statistics 17.0 software. $\mathrm{p}<0.05$ was considered statistically significant.

\section{Results}

\section{Characterization of Rat MSCs}

Rat MSCs had the capacity to adhere to plastic flasks in culture, and morphologically they appeared as spindle-shaped cells both as scattered individuals and in small colonies. After 3 passages, red blood cells were seldom seen by microscopy. Immunophenotype analysis indicated that the cultured cells had high levels of expression of CD44 (99.98\%), CD29 (100\%), and CD90 (99.79\%) and low expression of CD34 (5.04\%), CD45 (7.34\%), and CD11b (4.19\%) (fig. 1).

BM MSCs Transiently Retard Osteoblast Differentiation

\section{Characterization of Rat Osteoblasts}

Osteoblasts are usually isolated from newborn animals to ensure the cells have optimum osteogenic potential. Mineralization of the cells is an important parameter for identification. In the present study, rat osteoblasts had a triangular, spindle-like, or polygonal shape. After 3 days of culture, the cells grew exponentially, developing a large size and abundant cytoplasm. After 7-10 days of culture, the monolayer appeared to form a pavement structure. ALP staining of third passage cells showed that more than $90 \%$ of the osteoblasts stained red. The Alizarin red staining test showed calcium nodules which were oval shaped and stained red (fig. 2).

\section{The Effect of MSC-CM on Osteoblast Proliferation}

From day 1 to day 6, all groups showed a comparable increase in MTT activity (fig. 3a). There were significant differences in MTT activity after 2, 3, 4, and 6 days of culture between the 1:4 group (1 part MSC-CM:4 parts DMEM $+10 \%$ FBS) and the control group (DMEM + 10\% FBS only) ( $<<0.05)$. There were also significant differences in MTT activity after 2, 3, 4, 5, and 6 days of culture between the 1:2 group and the control group, and between the $1: 1$ group and the control group ( $<<0.05)$. These results indicated that MSC-CM suppresses osteoblast proliferation (fig. 3a).

To investigate whether the inhibitory effect of the MSC-CM was due to cell cycle alteration, osteoblasts cultured in various proportions of MSC-CM for 3 days were analyzed by flow cytometry. The proportion of cells in the $\mathrm{G} 2 / \mathrm{M}$ phase and the $S$ phase in the $1: 1$ group and the 1:2 group was significantly less than that in the control group ( $\mathrm{p}<0.05$ ), while the proportion of cells in the G2/M phase and the $S$ phase in the 1:4 group was not significantly different from that in the control group ( $\mathrm{p}>$ 0.05; fig. 3b, c).

\section{The Effect of MSC-CM on ALP Activity and Gene Expression}

The results of ALP activity assays are shown in figure 4a. Significantly lower ALP activity was observed in the $1: 3,1: 2$, and 1:1 MSC-CM groups compared with the control group $(p<0.05)$ after 3 days of culture. Furthermore, the lowest ALP activity was in the $1: 1$ group; it was significantly lower than in the $1: 3$ group $(\mathrm{p}<0.05)$ and not significantly different from that in the 1:2 group (fig. 4a).

Real-time PCR for the detection of ALP expression in osteoblasts was carried out after 3 days of culture in varied concentrations of MSC-CM. The results showed that 

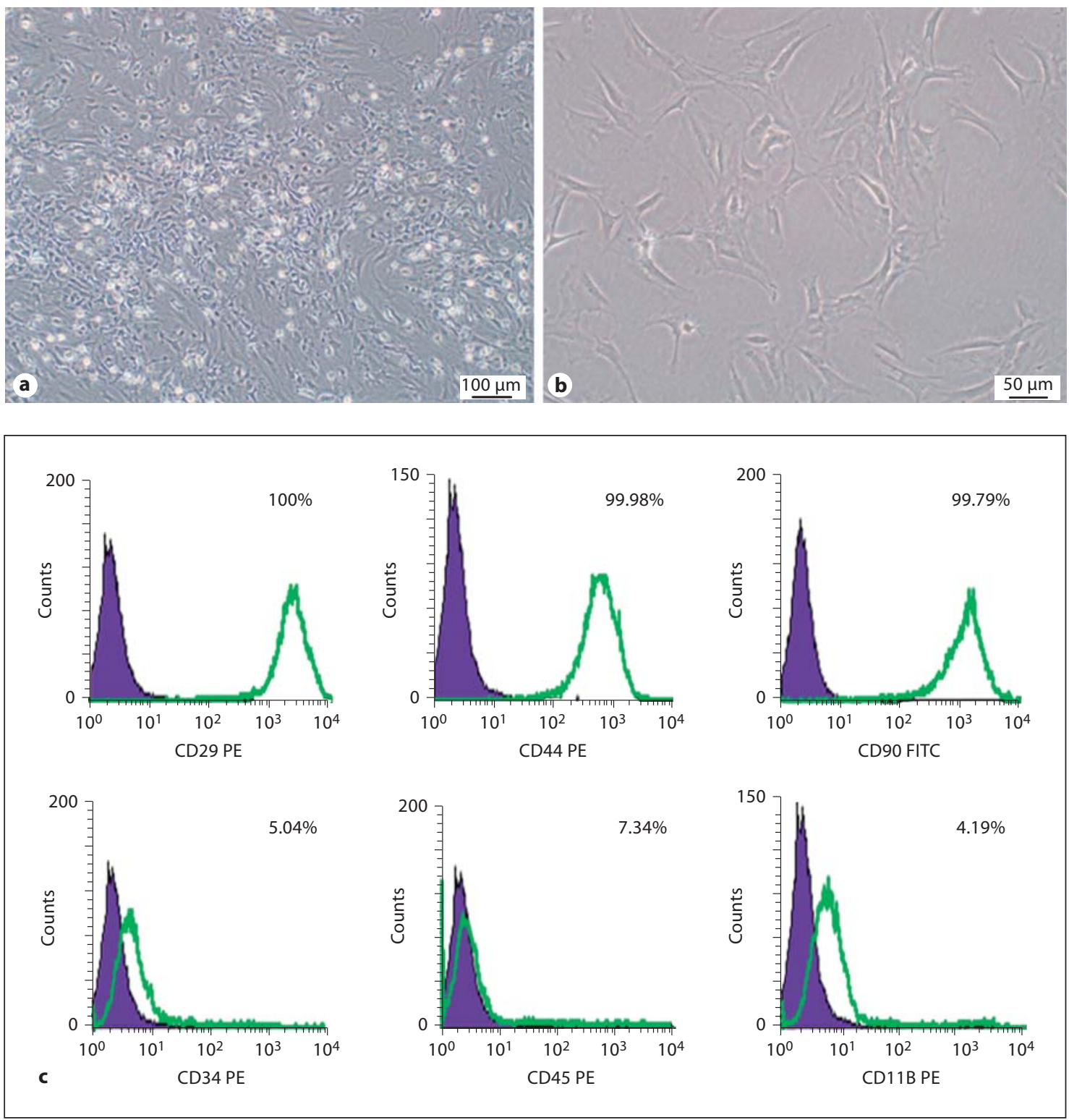

Fig. 1. Culture and identification of rat MSCs. a Primary MSCs in culture, 7 days after seeding. b MSCs of passage 3 in culture, 1 day after seeding. c Expression of CD29, CD44, CD90, CD34, CD45, and CD11b by MSCs at passage 3. Numbers indicate the percentages of cells positive for each of the surface markers.

ALP mRNA decreased when the cells were cultured in each MSC-CM compared to DMEM alone ( $\mathrm{p}<0.05$ ). Meanwhile, as shown in figure $4 \mathrm{~b}$, the ALP mRNA levels were associated with the concentrations of MSC-CM. A significant difference was found between any two MSCCM groups ( $\mathrm{p}<0.05)$, except between the $1: 1$ group and the $1: 2$ group $(\mathrm{p}>0.05)$. The ALP mRNA level was lowest in the 1:1 group, in which it was only a fifth of the control level (fig. 4b).
The Effect of MSC-CM on Osteoblastic Markers

Real-time PCR for the detection of OCN expression in osteoblasts was carried out after 3 days of culture in varied concentrations of MSC-CM. The results showed that MSC-CM also remarkably decreased the expression of OCN. OCN gene expression decreased when the cells were cultured in each MSC-CM compared to DMEM alone $(\mathrm{p}<0.05)$. Meanwhile, the OCN mRNA levels were also associated with the concentrations of MSC-CM. A 

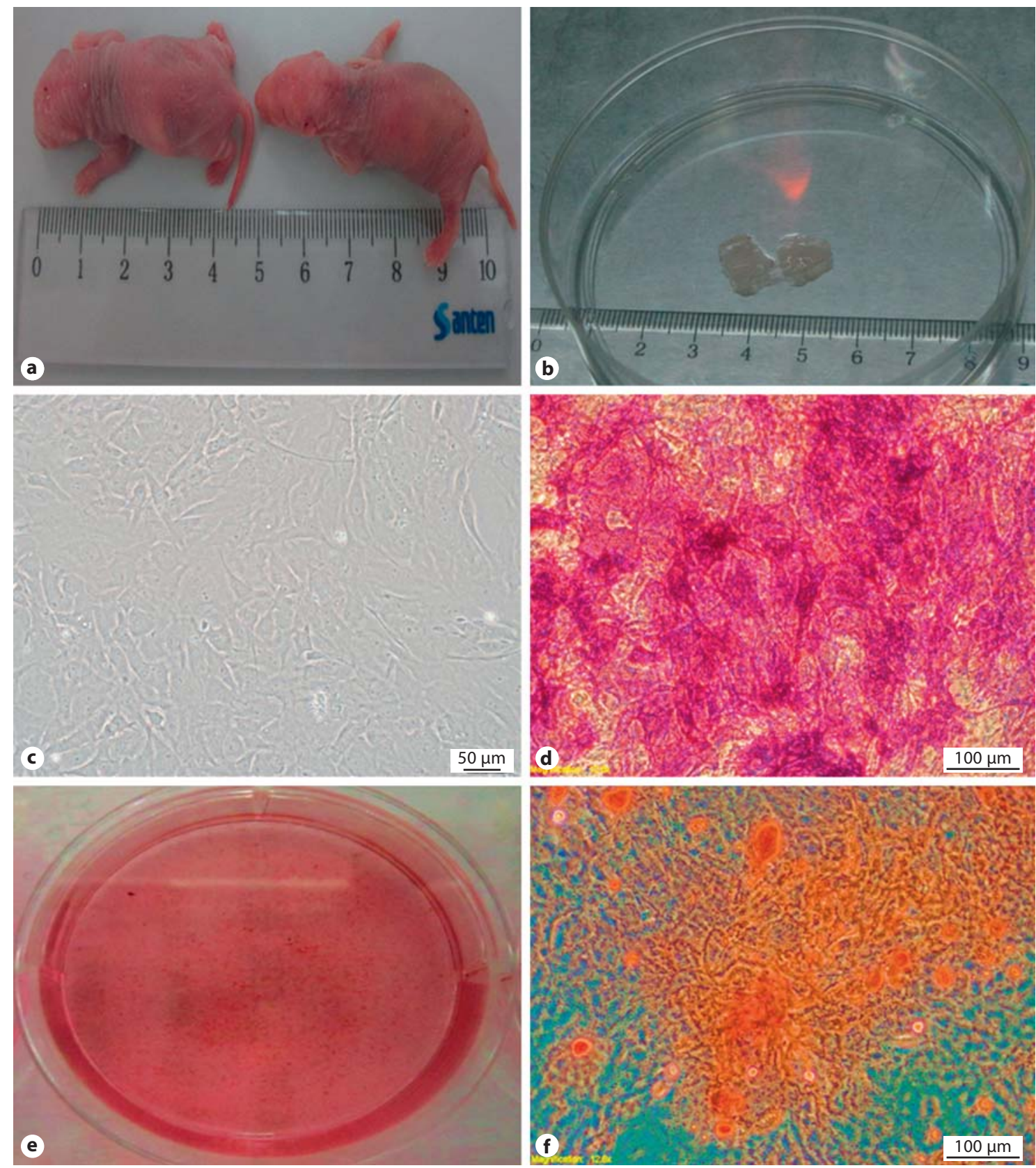

Fig. 2. Culture and identification of rat osteoblasts. a SD rats 24$48 \mathrm{~h}$ after birth. b Dissected calvariae of SD rats, with the periosteum completely stripped away. c Osteoblasts of passage 3 in culture, 4 days after seeding. d ALP staining of rat osteoblasts. Posi- tive cells were stained red. e Osteoblasts were subjected to Alizarin red S staining after culture in osteogenic induction medium for 14 days, seeded in a 6-cell plate. $\mathbf{f}$ Alizarin red S staining. Calcium nodules were oval shaped and stained red. significant difference was found between any two MSCCM groups ( $\mathrm{p}<0.05)$, except between the $1: 1$ group and the 1:2 group ( $\mathrm{p}>0.05)$. Again, the lowest level of OCN mRNA was observed in the 1:1 group (fig. 4c).
Western blotting of OCN and OPN was performed to detect the protein expression of osteoblastic markers. Decreased osteoblastic protein expression was observed in each of the MSC-CM groups compared with the control 


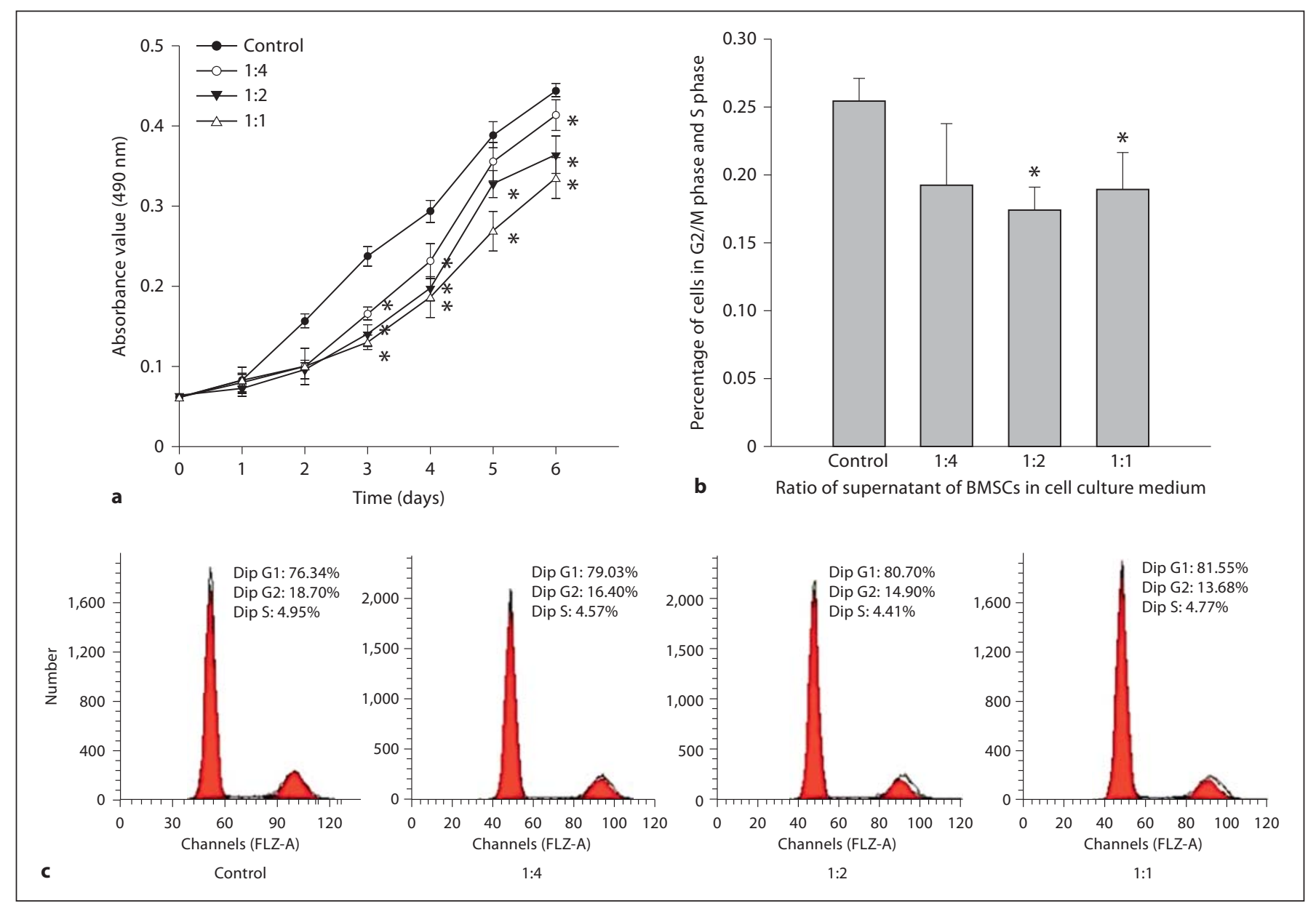

Fig. 3. The effect of MSC-CM on the proliferation and cell cycle progression of osteoblasts. a Cell proliferation curves of osteoblasts by MTT assay. The proliferation of the cells was not significantly different between the MSC-CM groups and the control group for 1-2 days. The absorbance in the 1:1 and 1:2 groups was significantly lower than in the control group from 3 to 6 days. $\left({ }^{*} \mathrm{p}<0.05 ; \mathrm{n}=6\right)$. The absorbance in the $1: 4$ group was signifi- cantly lower than in the control group on days 3,4 and $6 .\left(^{*} \mathrm{p}<\right.$ $0.05 ; \mathrm{n}=6)$. $\mathbf{b}$ Flow cytometry determined the change in the cell cycle after co-culture for 3 days. c Histogram showing the percentage of cells in the G2/M phase and in the $S$ phase in each culture group. Compared with the control group, the percentage of cells in $\mathrm{G} 2 / \mathrm{M}$ and $\mathrm{S}$ phase was significantly lower in the 1:2 and $1: 1$ groups $(\mathrm{p}<0.05)\left({ }^{*} \mathrm{p}<0.05, \mathrm{n}=3\right)$. group $(\mathrm{p}<0.05)$. There was only about a half of OPN or OCN expression in samples of the 1:1 group (fig. $4 \mathrm{~d}$, e).

Immunofluorescent staining of OCN was also performed. Almost all cells in the control group were OCN positive. There were fewer OCN-positive cells in the 1:3 group than in the control group, and the least OCN-positive cells were in the 1:1 group (fig. 7a).

\section{The Effect of MSC-CM on Osteoblastic Transcription Factors}

As a result of the data on the osteoblastic markers, Western blot and real-time PCR of Runx2 and Osx was performed to detect any effects of MSC-CM on the gene expression of osteoblastic transcription factors. Decreased Runx2 expression was observed at both mRNA and protein levels in each of the MSC-CM groups compared with the control group ( $\mathrm{p}<0.05)$. In addition, the decreased effect was concentration dependent. The higher the concentration of MSC-CM was, the lower the Runx-2 level was. Interestingly, the expression of Osx showed no noticeable difference among the different groups ( $\mathrm{p}>0.05$; fig. 5).

Results of immunofluorescent staining of Runx2 showed many Runx2-positive cells in the control group, 


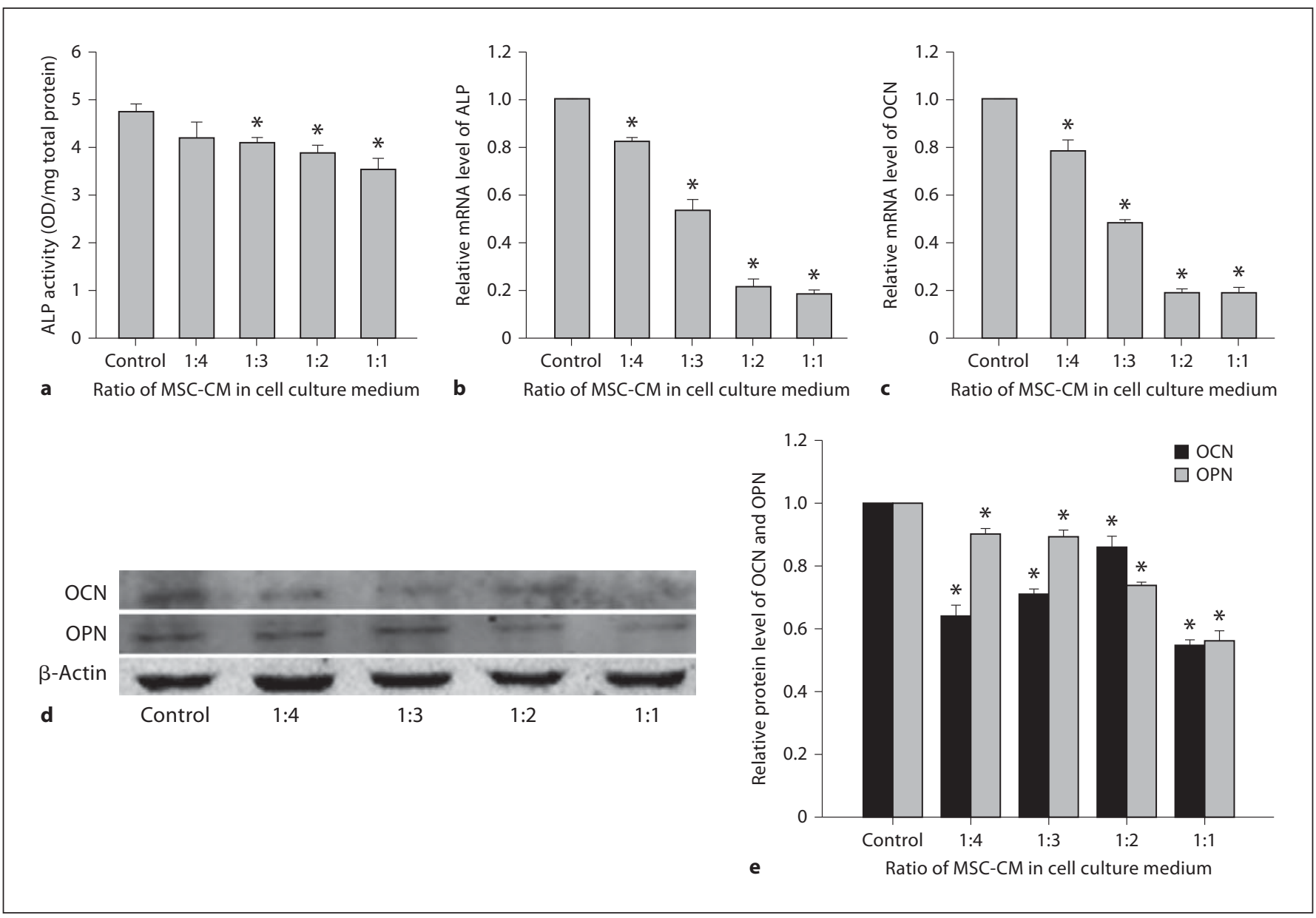

Fig. 4. Effect of MSC-CM on the expression of osteoblastic markers at 3 days. a ALP activity of osteoblasts in all groups after 3 days of culture. There was a significant difference between the 1:2 and 1:1 MSC-CM groups with the control group $(\mathrm{p}<0.05)$. Data are presented as means \pm standard deviations $(n=4$ per group) ( $\left.{ }^{*} \mathrm{p}<0.05\right)$. b ALP mRNA expression level on day 3 , analyzed by real-time RT-PCR and normalized against GAPDH. Compared with the control group, the mRNA expression level was significantly lower in each of the MSC-CM groups $(\mathrm{p}<0.05)$. The 1:1 group showed the lowest level of ALP mRNA expression. Data are presented as means \pm standard deviations $(n=4$ per

fewer positive cells in the 1:3 group, and the least positive cells in the 1:1 group (fig. 7b).

\section{The Effect of MSC-CM on Osteoblastic Molecules after 7 Days}

To detect whether osteoblast differentiation was actually reduced or just delayed in comparison to untreated cells, Western blot and real-time PCR of osteoblastic molecules was performed after 7 days of treatment. The levels group) (* p < 0.05). c OCN mRNA expression level on day 3 , analyzed by real-time RT-PCR and also normalized against GAPDH. Compared with the control group, the mRNA expression level was significantly lower in each of the MSC-CM groups $(\mathrm{p}<0.05)$. The 1:1 group showed the lowest level of OCN mRNA expression. Data are presented as means \pm standard deviations ( $\mathrm{n}=4$ per group) $\left({ }^{*} \mathrm{p}<0.05\right)$. $\mathbf{d}$ Western blot analysis of OPN and OCN. $\beta$-Actin was used as the internal control. e Quantification of the protein levels showed an decrease in OCN and OPN protein levels in 1:4, 1:3, 1:2, and 1:1 MSC-CM groups $\left({ }^{*} \mathrm{p}<0.05\right)$ $(\mathrm{n}=3)$. of OCN, OPN, and Runx2 of cells in CM groups went back to normal compared with cells in the control group. Expression of these three molecules showed no significant difference among groups ( $p>0.05$; fig. 6$)$.

Immunofluorescent staining of OCN and Runx 2 was performed on cells treated for 7 days with CM. Figure 7c, $\mathrm{d}$ shows that cells in each group were almost all stained positive. No significant difference was observed among groups (fig. 7c, d). 

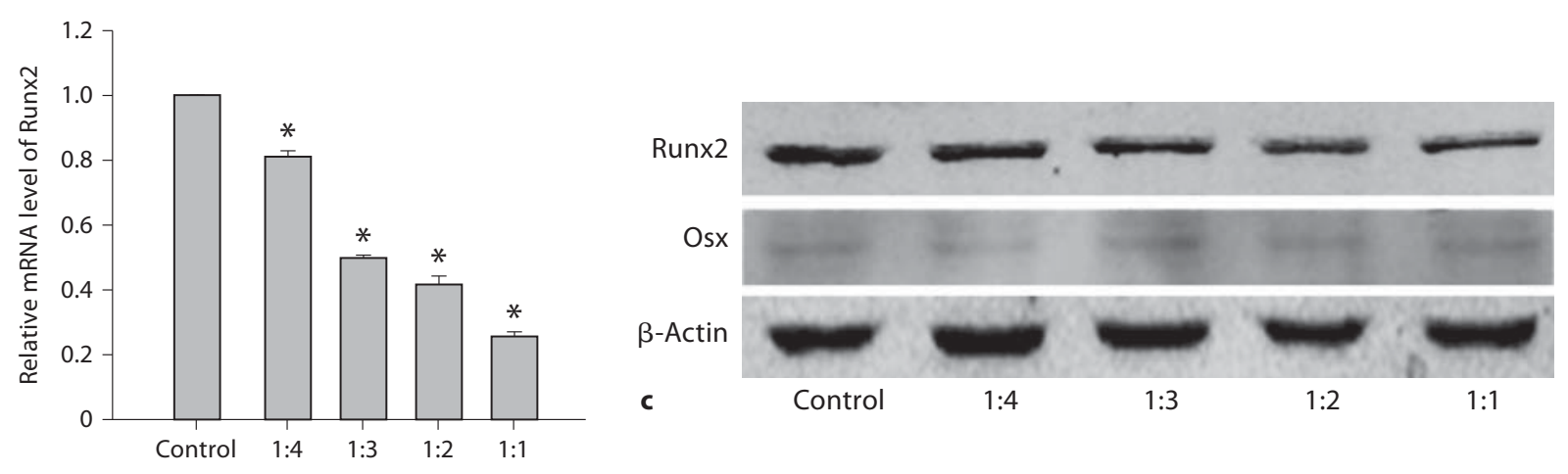

a

Ratio of MSC-CM in cell culture medium
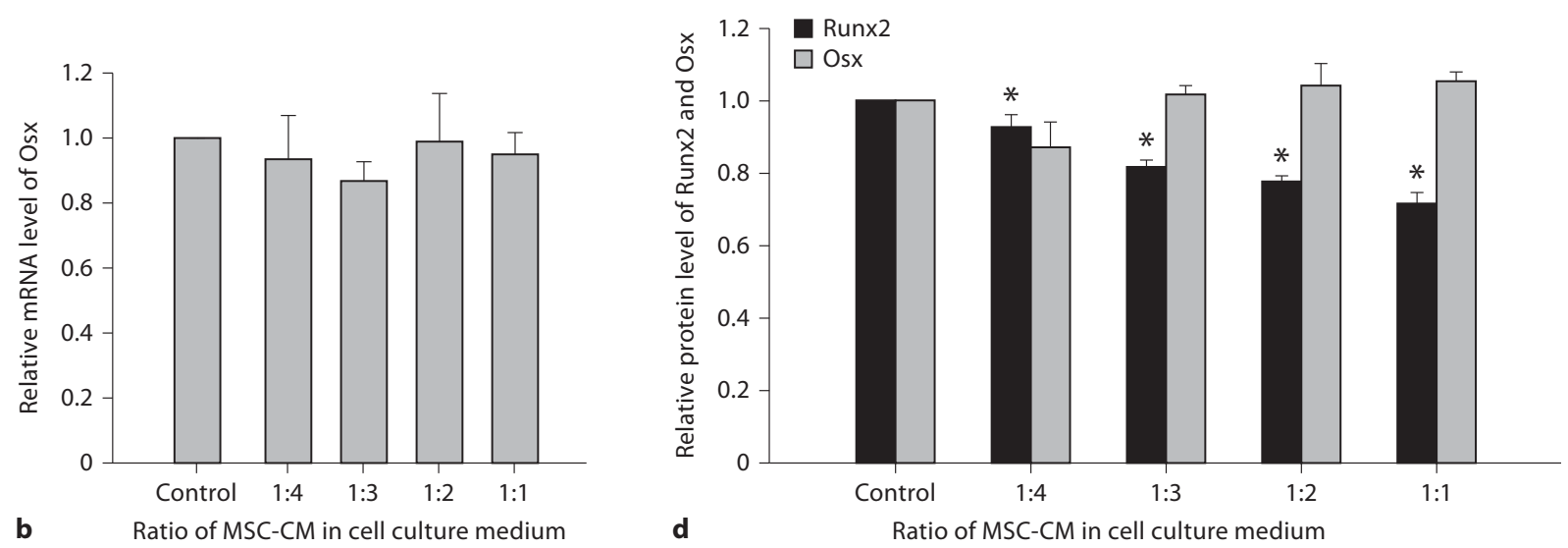

Fig. 5. Effect of MSC-CM on the expression of osteoblastic transcription factors at 3 days. a Runx 2 mRNA expression level on day 3 analyzed by real-time RT-PCR and normalized against GAPDH. Compared with the control group, Runx2 mRNA expression was significantly lower in each of the MSC-CM groups $(\mathrm{p}<0.05)$. The 1:1 group showed the lowest Runx 2 mRNA expression. Data are presented as means \pm standard deviations $(\mathrm{n}=4$ per group) $\left({ }^{*} \mathrm{p}<0.05\right)$. b Osx mRNA expression level on day 3 analyzed by real-time RT-PCR and normalized against GAPDH.
Expression of Osx showed no noticeable difference among different groups $(\mathrm{p}>0.05)$. Data are presented as means \pm standard deviation ( $n=4$ per group). c Western blot analysis of Runx 2 and Osx. $\beta$-Actin was used as the internal control. d Quantification of the protein levels showed an obvious decrease in the Runx2 protein level in the $1: 4,1: 3,1: 2$, and 1:1 MSC-CM groups ( $\mathrm{p}<$ $0.05)$. No change could be detected in the Osx protein level among the groups $(\mathrm{p}>0.05)\left({ }^{*} \mathrm{p}<0.05, \mathrm{n}=3\right)$.

in the $\mathrm{G} 2 / \mathrm{M}$ and $\mathrm{S}$ phase. It has been reported that MSCs could inhibit the proliferation of Th1 cells and prevent dendritic cells from entering the cell cycle by downregulating proteins involved in the cell cycle [Aggarwal and Pittenger 2005; Ramasamy et al., 2007]. In fact, many cytokines may play a part in the effect of MSCs on cell proliferation, such as interleukins and prostaglandins [Spaggiari et al., 2009]. These factors may play the same roles in controlling the proliferation of osteoblasts, as they are very sensitive to the microenvironment. There is abundant evidence that the mesenchyme-derived microenvironment affects not only the differentiation of hemopoi- 




Fig. 6. Effect of MSC-CM on the expression of osteoblastic markers and osteoblastic transcription factors at 7 days. a OCN mRNA expression level on day 7 analyzed by real-time RT-PCR and normalized against GAPDH. Expression of OCN showed no significant difference among different groups $(\mathrm{p}>0.05)$. Data are presented as means \pm standard deviations ( $\mathrm{n}=4$ per group). $\mathbf{b}$ Runx 2 mRNA expression level on day 7 analyzed by real-time RT-PCR and normalized against GAPDH. Expression of Runx2 showed no significant difference among different groups ( $\mathrm{p}>0.05)$. Data are presented as means \pm standard deviations ( $\mathrm{n}=4$ per group). c Western blot analysis of OCN, OPN, and Runx2. $\beta$-Actin was used as the internal control. d Quantification of the protein levels showed no significant difference among the groups $(\mathrm{p}>0.05$, $\mathrm{n}=3$ ). etic cells but also the proliferation and function of many other cells through different signaling pathways.

For osteoblast differentiation investigation, we detected the ALP level, which is involved in the mineralization process and is the most widely recognized marker of osteogenesis. ALP levels usually increase during the early stages of osteoblast differentiation. To date, it has been reported that either mechanical or biochemical signals might influence the proliferation and differentiation of osteoblasts [Kadow-Romacker et al., 2009; Fei et al., 2011]. There are no previous reports that MSCs had effects on the activation and differentiation of osteoblasts. In this paper, using ALP assay, we first obtained the result that MSC-CM transiently suppresses osteoblast differentiation and leads to a reduction in ALP. We did not repeat the ALP detection on day 7 because it was not in the early stage of osteoblast differentiation yet.

OCN is a major osteoblast-related gene product synthesized only by mature osteoblasts. Expression of OCN is an important indicator of the osteoblastic differentiation of osteoblasts. OPN is a highly expressed gene in bone, and increased levels of OPN may indicate osteo- 

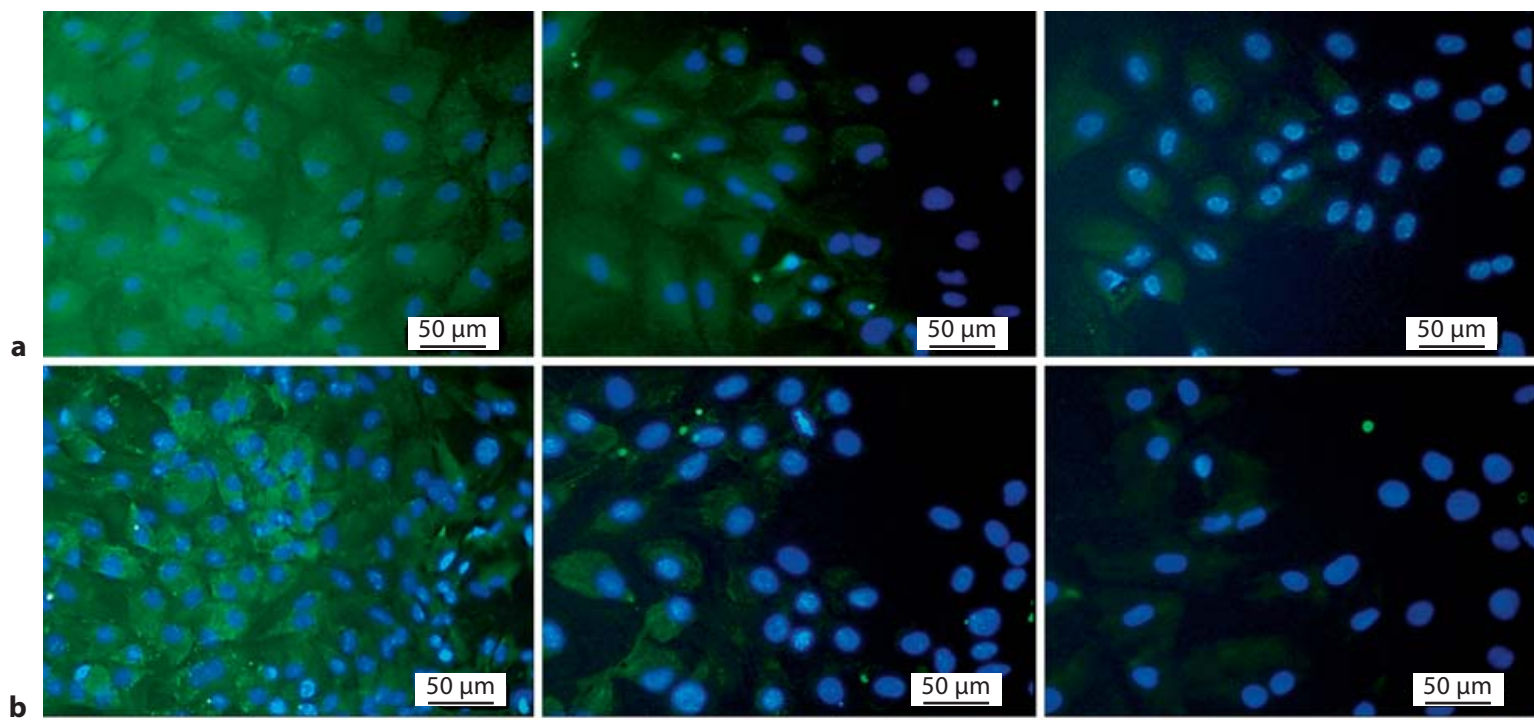

b
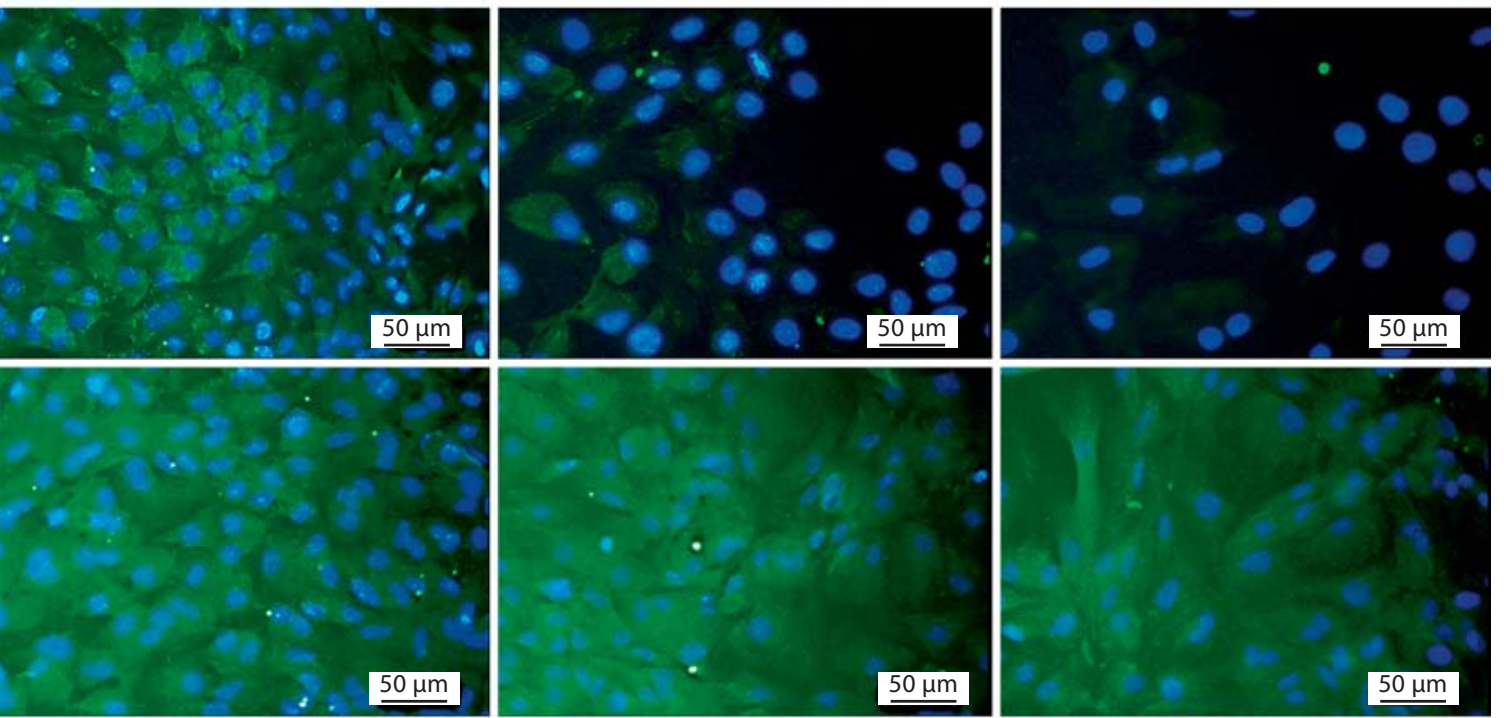

C

$\underline{50 \mu \mathrm{m}}$

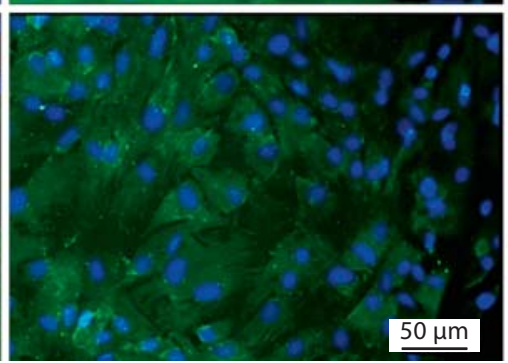

$1: 3$

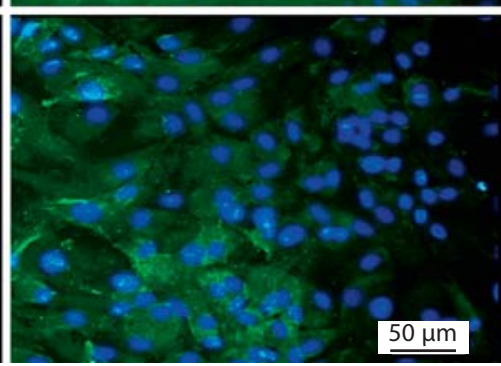

$1: 1$

Fig. 7. Immunofluorescent analysis of osteoblastic molecules OCN and Runx2. a Immunofluorescent analysis of OCN on day 3. There were fewer OCN-positive cells in CM groups than in the control group. b Immunofluorescent analysis of Runx 2 on day 3 . There were fewer Runx2-positive cells in CM groups than in the control group. c Immunofluorescent analysis of OCN on day 7. Almost all cells in each group were OCN-positive stained. d Immunofluorescent analysis of Runx 2 on day 7. Almost all cells in each group were Runx2-positive stained. Blue represents the nuclei; green represents OCN/Runx2. blast differentiation [Frank et al., 2002]. As the results show, OPN and OCN were significantly decreased by MSC-CM compared to the control group on day 3 and went back to normal on day 7. MSCs may therefore transiently retard osteoblast differentiation.

In order to evaluate the molecular mechanisms underlying this transient osteogenic suppression, levels of two important osteoblastic transcription factors, Runx 2 and
Osx, were measured at both mRNA and protein levels. Runx-2 plays a crucial role in osteoblastic differentiation and is essential to normal osteogenesis. During osteoblast maturation, it is usually upregulated at first. Runx-2 deficiency or mutation causes severe bone abnormalities [Pratap et al., 2003; Chen et al., 2011]. In the present study, both the mRNA and protein level of Runx-2 were decreased by MSC-CM on day 3. MSCs were thus able to 
inhibit the osteoblastic differentiation of osteoblasts. Osx is usually considered an inhibitor of chondrogenesis and chondrocyte maturation, thus promoting osteoblast maturation [Nakashima et al., 2002]. The present results suggest that Osx might not be inhibited by MSCs. This is a very interesting finding and suggests that although MSCs transiently retard differentiation of osteoblasts they do not promote chondrogenesis differentiation of the cells.

Previous reports have shown that several bioactive factors, such as fibroblast growth factor (FGF-2), bone morphogenetic proteins (BMPs), IL-6, TNF- $\alpha$, msh homeobox 2 (Msx2), and oligodeoxynucleotide, could modulate osteoblast differentiation and bone formation [Javed et al., 2009; Lee et al., 2010; Fei et al., 2011; Feng et al., 2011]. IL- 6 can promote osteoblast differentiation by increasing the expression of osteogenic markers ALP and OCN [Taguchi et al., 1998]. It may also exert effects on osteoblast differentiation indirectly by controlling the local production of insulin growth factor 1 (IGF-1), BMP-4, and BMP6 [Franchimont et al., 1997; Yeh et al., 2002]. TNF- $\alpha$ decreases osteoblastic bone formation through the promotion of cell apoptosis, a decrease in osteoblast differentiation, and the suppression of ALP activity and OCN expression [Kuno et al., 1994; Kitajimaa et al., 1996; Gilbert et al., 2002]. It has also been reported that MSCs may inhibit protein production and gene expression of both IL- 6 and TNF- $\alpha$ [Guo et al., 2007]. Maybe the transient osteoblast differentiation retardation observed in our study is a combined effect of IL- 6 and TNF- $\alpha$. Other cytokines such as IGF and BMPs may also be involved in the mechanism. MSCs may secrete several cytokines, and these mediators interact with each other.

In this paper, we demonstrated that MSCs may have a significant effect on the suppression of osteoblast proliferation and transient retardation of osteoblast differentiation. This study highlights the need to take into account the paracrine effect of MSCs when using them in regenerative medicine for the repair of bone defects.

\section{Acknowledgments}

The research was supported by the Shanghai Leading Academic Discipline Project (S30205), the National Natural Science Foundation of China (81000404 and 81100696), and the Young Talents Training Program of the Shanghai Health Bureau (XYQ2011053).

\section{References}

Aggarwal, S., M.F. Pittenger (2005) Human mesenchymal stem cells modulate allogeneic immune cell responses. Blood 105: 1815-1822.

Caplan, A.I. (2007) Adult mesenchymal stem cells for tissue engineering versus regenerative medicine. J Cell Physiol 213: 341-347.

-Caplan, A.I, J.E. Dennis (2006) Mesenchymal stem cells as trophic mediators. J Cell Biochem 98: 1076-1084

Chen, H., F.Y. Ghori-Javed, et al (2011) Chondrocyte-specific regulatory activity of runx 2 is essential for survival and skeletal development. Cells Tissues Organs 194: 161-165.

Chun-mao, H., W. Su-yi, et al (2007) Human bone marrow-derived mesenchymal stem cells differentiate into epidermal-like cells in vitro. Differentiation 75: 292-298.

Deans, R.J., A.B. Moseley (2000) Mesenchymal stem cells: biology and potential clinical uses. Exp Hematol 28: 875-884.

Erices, A., P. Conget, et al (2000) Mesenchymal progenitor cells in human umbilical cord blood. Br J Haematol 109: 235-242.

Fei, Y., L. Xiao, et al (2011) FGF2 stimulation of osteoblast differentiation and bone formation is mediated by modulation of the WNT pathway. J Biol Chem 286: 40575-40583.
Feng, Z., Y. Shen, et al (2011) An oligodeoxynucleotide with promising modulation activity for the proliferation and activation of osteoblast. Int J Mol Sci 12: 2543-2555.

Franchimont, N., V. Gangji, et al (1997) Interleukin- 6 with its soluble receptor enhances the expression of insulin-like growth factor-I in osteoblasts. Endocrinology 138: 5248-5255.

Frank, O., M. Heim, et al (2002) Real-time quantitative RT-PCR analysis of human bone marrow stromal cells during osteogenic differentiation in vitro. J Cell Biochem 85: 737746.

Gilbert, L., X. He, et al (2002) Expression of the osteoblast differentiation factor RUNX2 (Cbfa1/AML3/Pebp2alpha A) is inhibited by tumor necrosis factor-alpha. J Biol Chem 277: 2695-2701.

Gronthos, S., D.M. Franklin, et al (2001) Surface protein characterization of human adipose tissue-derived stromal cells. J Cell Physiol 189: 54-63.

Guo, J., G.S. Lin, et al (2007) Anti-inflammation role for mesenchymal stem cells transplantation in myocardial infarction. Inflammation 30: 97-104.
Guo, Z., C. Zheng, et al (2009) Fetal BM-derived mesenchymal stem cells promote the expansion of human Th17 cells, but inhibit the production of Th1 cells. Eur J Immunol 39: 2840-2849.

Hass, R., C. Kasper, et al (2011) Different populations and sources of human mesenchymal stem cells (MSC): a comparison of adult and neonatal tissue-derived MSC. Cell Commun Signal 9: 12 .

Horwitz, E.M. (2006) MSC: a coming of age in regenerative medicine. Cytotherapy 8: 194195.

Javed, A., F. Afzal, et al (2009) Specific residues of RUNX2 are obligatory for formation of BMP2-induced RUNX2-SMAD complex to promote osteoblast differentiation. Cells Tissues Organs 189: 133-137.

Kadow-Romacker, A., J.E. Hoffmann, et al (2009) Effect of mechanical stimulation on osteoblast- and osteoclast-like cells in vitro. Cells Tissues Organs 190: 61-68.

Kasten, P., J. Vogel, et al (2006) Influence of platelet-rich plasma on osteogenic differentiation of mesenchymal stem cells and ectopic bone formation in calcium phosphate ceramics. Cells Tissues Organs 183: 68-79. 
Kitajima, I., Y. Soejima, et al (1996) Ceramideinduced nuclear translocation of NF-kappa $B$ is a potential mediator of the apoptotic response to TNF-alpha in murine clonal osteoblasts. Bone 19: 263-270.

Kuno, H., S.M. Kurian, et al (1994) Inhibition of 1,25-dihydroxyvitamin D3 stimulated osteocalcin gene transcription by tumor necrosis factor-alpha: structural determinants within the vitamin D response element. Endocrinology 134: 2524-2531.

Lee, H. L., T. Yi, et al (2010) Msx2 mediates the inhibitory action of TNF-alpha on osteoblast differentiation. Exp Mol Med 42: 437445.

Majumdar, M.K., M. Keane-Moore, et al (2003) Characterization and functionality of cell surface molecules on human mesenchymal stem cells. J Biomed Sci 10: 228-241.

Majumdar, M.K., M.A. Thiede, et al (2000) Human marrow-derived mesenchymal stem cells (MSCs) express hematopoietic cytokines and support long-term hematopoiesis when differentiated toward stromal and osteogenic lineages. J Hematother Stem Cell Res 9: 841-848.

Nakashima, K., X. Zhou, et al (2002) The novel zinc finger-containing transcription factor osterix is required for osteoblast differentiation and bone formation. Cell 108: 17-29.
Pittenger, M.F., A.M. Mackay, et al (1999) Multilineage potential of adult human mesenchymal stem cells. Science 284: 143-147.

Pratap, J., M. Galindo, et al (2003) Cell growth regulatory role of Runx 2 during proliferative expansion of preosteoblasts. Cancer Res 63: 5357-5362.

Ramasamy, R., H. Fazekasova, et al (2007) Mesenchymal stem cells inhibit dendritic cell differentiation and function by preventing entry into the cell cycle. Transplantation 83 : 71-76.

Spaggiari, G.M., H. Abdelrazik, et al (2009) MSCs inhibit monocyte-derived DC maturation and function by selectively interfering with the generation of immature DCs: central role of MSC-derived prostaglandin E2. Blood 113: 6576-6583.

Sun, H., C. Wu, et al (2006) Proliferation and osteoblastic differentiation of human bone marrow-derived stromal cells on akermanite-bioactive ceramics. Biomaterials 27: 5651-5657.
Taguchi, Y., M. Yamamoto, et al (1998) Interleukin-6-type cytokines stimulate mesenchymal progenitor differentiation toward the osteoblastic lineage. Proc Assoc Am Physicians 110: 559-574.

Xiao, C., H. Zhou, et al (2011) Bone marrow stromal cells with a combined expression of BMP-2 and VEGF-165 enhanced bone regeneration. Biomed Mater 6: 015013.

Yeh, L.C., M.C. Zavala, et al (2002) Osteogenic protein-1 and interleukin- 6 with its soluble receptor synergistically stimulate rat osteoblastic cell differentiation. J Cell Physiol 190: 322-331.

Zhang, J., S. Huang, et al (2010) Targeted knockdown of Bcl2 in tumor cells using a synthetic TRAIL 3'-UTR microRNA. Int J Cancer 126: 2229-2239.

Zhang, H., B. Niu, et al (2011) Interruption of intrachromosomal looping by CCCTC binding factor decoy proteins abrogates genomic imprinting of human insulin-like growth factor II. J Cell Biol 193: 475-487.

- Zhou, H., C. Xiao, et al (2011) In vivo efficacy of bone marrow stromal cells coated with betatricalcium phosphate for the reconstruction of orbital defects in canines. Invest Ophthalmol Vis Sci 52: 1735-1741. 